\title{
Gayet-Wernicke Encephalopathy: 2 Case Reports
}

\author{
Y. EL Badri ${ }^{1 *}$, S Agharas ${ }^{1}$, S. Alj ${ }^{1}$, M. Ouali Idrissi ${ }^{2}$, N. Cherif Idrissi Ganouni ${ }^{2}$, H. Rebahi $^{3}$
}

\author{
${ }^{1}$ Radiology department, Ibn Tofail hospital, Mohammed VI university hospital, Cadi Ayyad University, Marrakech, Morocco \\ ${ }^{2}$ Radiology department, Arrazi hospital, Mohammed VI University hospital, Cadi Ayyad University, Marrakech, Morocco \\ ${ }^{3}$ Maternity intensive care unit, Ibn Tofail Hospital, Mohammed VI University Hospital, Cadi Ayyad University, Marrakech, Morocco
}

DOI: $10.36347 /$ sjmcr.2021.v09i03.030

| Received: 23.02.2021 | Accepted: 08.03.2021 | Published: 30.03.2021

*Corresponding author: Y. EL Badri

Abstract

Gayet-Wernicke encephalopathy is an acute neurological complication due to thiamine deficiency. The diagnosis can be difficult to make since the clinical forms can be poor or atypical. Although it is often associated with chronic alcoholism, it can also occur in all the conditions that lead to thiamine deficiency: undernutrition, chronic vomiting, prolonged fasting, and exclusive artificial feeding. It is a rare complication, reversible if is promptly treated. We report two cases of Gayet-Wernicke encephalopathy, the first occurred in a pregnant patient suffering from uncontrollable vomiting and the second patient treated with methotrexate for rheumatoid arthritis with a chronic uncontrollable vomiting due to digestive intolerance to Methotrexate (only cause found).

Keywords: Gayet-Wernicke encephalopathy, MRI, medical emergency.

Copyright $(\odot) 2021$ The Author(s): This is an open-access article distributed under the terms of the Creative Commons Attribution 4.0 International License (CC BY-NC 4.0) which permits unrestricted use, distribution, and reproduction in any medium for non-commercial use provided the original author and source are credited.

\section{INTRODUCTION}

Gayet-Wernicke encephalopathy is an acute neuropsychiatric complication caused by thiamine (vitamin B1) deficiency. It is frequently seen in heavy drinkers, but can sometimes be seen in other circumstances. Clinically, Gayet-Wernicke encephalopathy is manifested by a classic very evocative triad associating consciousness disorders ranging from temporo-spatial disorientation to coma, oculomotor disorders and ataxia. But this triad is only found in $10 \%$ of cases. Brain MRI is the modality of choice, it shows in classic forms T1 hypointense and T2 and Flair hyperintense lesions characteristic by their location.

We report two cases illustrating the MRI aspects found in this pathology with some not calssic etiologies.

\section{Case Number 1}

We report the case of a 28-year-old patient, pregnant at 16 weeks with amenorrhea, suffering from uncontrollable vomiting since the onset of pregnancy. She presented epigastric pain with a high level of lipasemia at $218 \mathrm{U} / \mathrm{L}$. The patient was therefore hospitalized for acute pancreatitis and for fluid and electrolyte disturbances. suddenly presented confusion, temporo-spatial disorientation and then troubles of consciousness. A brain MRI without Gadolinium injection was performed showing T2/Flair and diffusion hypersignal in per aqueductal, peri ventricular (V3), mammillary bodies and the two thalami without edema or mass effect, evoking Gayet Wernicke encephalopathy (fig 1).

The follow-up MRI five days after thiamine supplementation revealed a clear regression of the lesions, with clinically persistent temporo-spatial disorientation (fig 2). 

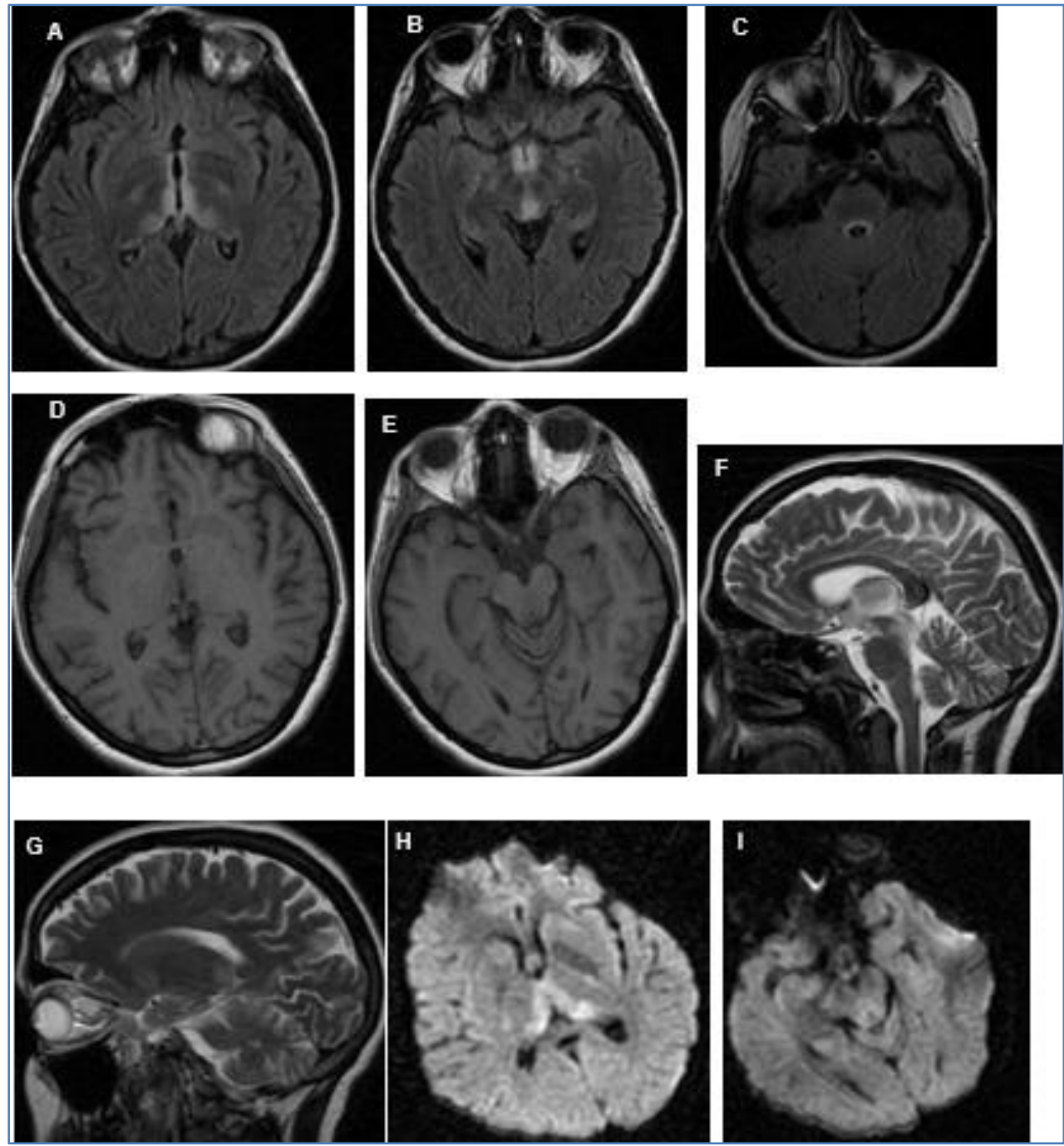

Fig-1: Brain MRI in FLAIR (A, B and C), T1 (D and E), T2 (F and G), and diffusion (H and I) sequences per aqueductal, peri ventricular (V3) , mammillary bodies and the two thalami lesions in iso signal T1, hyper signal T2, Flair and diffusion, without edema or mass effect.
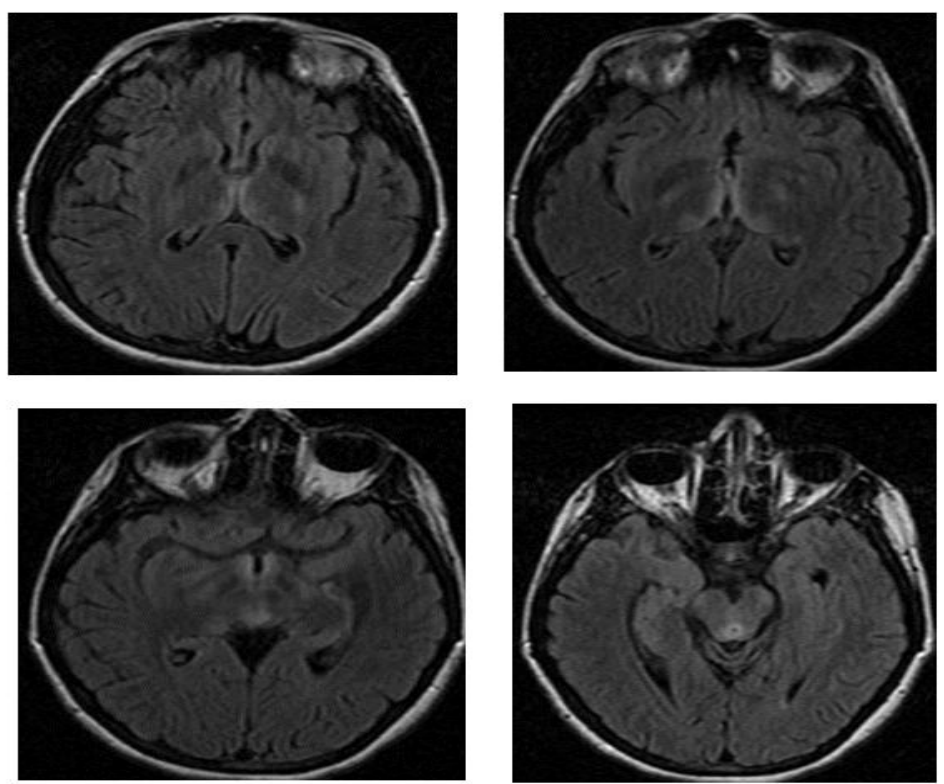

Figure 2: Brain MRI in Flair sequence in axial sections performed five days after thiamine supplementation, showing a regression of the lesions described above. 


\section{CASe Number 2}

We report the case of a 47-year-old patient, followed for infectious polyarthritis on rheumatoid arthritis, non-alcoholic, admitted for acute rotatory dizziness, bilateral tinnitus and horizontal nystagmus associated with severe digestive intolerance to Methotrexate revealed by postprandial incoercible vomiting complicated by Mallory Weiss syndrome. Brain MRI without Gadolinium injection revealed a bilateral and symmetrical Flair and T2 hypersignal of the two thalami, the mammillary bodies and in the peracqueductal region suspecting a Gayet Wernicke's encephalopathy (fig $3 \mathrm{~A}$ ).

Post-treatment control (10th day after supplentation) was satisfying with disappearance of clinical signs and regression of brain lesions (fig $3 \mathrm{~B}$ ).
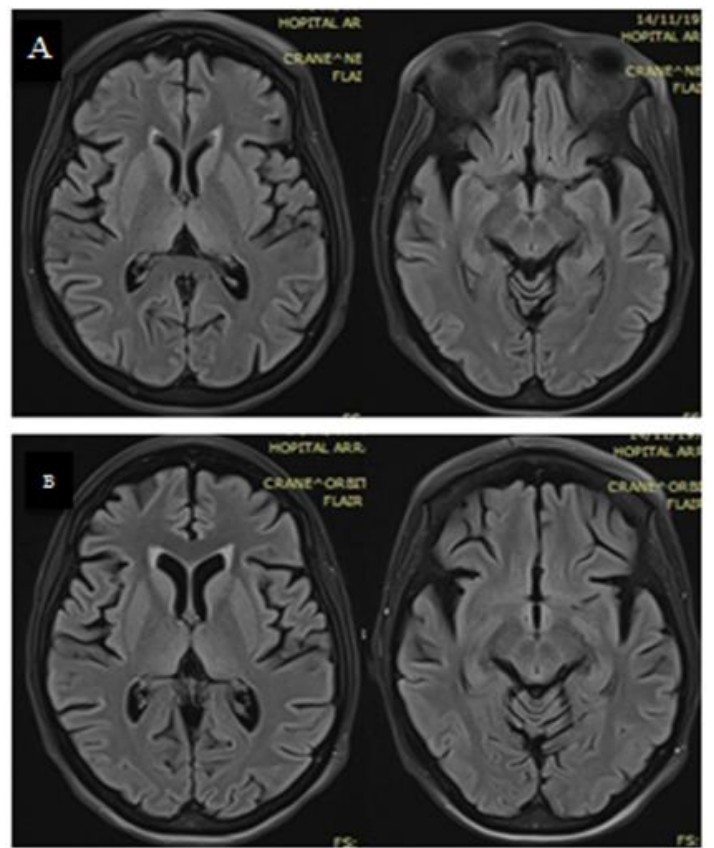

Fig-3: A: Brain MRI in axial Flair sequence showing a bilateral and symmetrical hypersignal of the two thalami and peracqueductal region. B: Brain MRI in axial Flair sequence after treatment showing regression of lesions

\section{Discussion}

Gayet-Wernicke encephalopathy is an acute neurological complication caused by thiamine (vitamin B1) deficiency, first described in 1881 by Carl Wernicke in an alcoholic man and a woman with uncontrollable vomiting [1]. It is a rare disease whose autopsy prevalence estimated at $0.8-2.8 \%$ is much higher than the prevalence observed with clinical manifestations $(0.04-0.13 \%)$. It is observed during chronic alcoholism, but more generally in a context of severe undernutrition: pregnancy vomiting, prolonged parenteral nutrition, hunger strike, gastrointestinal tumors, cancer and chemotherapy, digestive surgery, pyloric stenosis, terminal chronic renal failure, anorexia nervosa [1].
The daily thiamine requirements are of the order of $1.4 \mathrm{mg} /$ day and are increased by a high calorie diet or a diet rich in carbohydrates [2]. The thiamine deficiency causes lesions of varying severity in the brain, ranging from hemorrhagic suffusions to the destruction of neurons. Brain damage sets in within 2 to 3 weeks, the time required to deplete the body's stores being 18 days. The onset time of clinical signs is on average 4 weeks [3]. In the first case report, the uncontrollable vomiting since the beginning of the pregnancy was the main contributing factor, aggravated by the resting of the digestive tract as part of the management of acute pancreatitis.

For the second patient, she was on Methotrexate, which is the basic treatment for her rheumatoid arthritis, known for its often minor, nonlife-threatening digestive side effects. However, these effects can be very debilitating, requiring the interruption of treatment as in the case of our patient.

Clinically, Gayet-Wernicke encephalopathy is manifested by a very evocative classic triad associating consciousness disorders ranging from temporo-spatial disorientation to coma, oculomotor disorders (nystagmus and oculomotor paralysis) and ataxia, but found only in $10 \%$ of cases [4].

In $80 \%$ of cases, it is a non-specific confusional state of cognitive impairment, drowsiness, stupor or coma: this was the case with our first patient.

In $19 \%$ of cases, no signs are found. However, the diagnosis is very likely when two of the following four conditions are present: malnutrition, oculomotor disorders, cerebellar disorders, altered mental state or moderate memory impairment. The chronic form of Gayet Wernicke's encephalopathy produces Korsakoff syndrome characterized by memory disturbances, anasognosia, fabulations and false recognition.

Brain MRI is the exam of choice with a $53 \%$ sensitivity and a $93 \%$ specificity. In the classic forms, it shows lesions hypointense in T1 and hyperintense in T2 and Flair, characteristic by their location $[5,6]$ : bilateral and symmetrical involvement of the median thalami and the peri-ventricular regions of the third ventricle $(85 \%)$, involvement of the periaqueductal area (65\%), of the mammillary bodies $(58 \%)$. Other localizations are possible such as the tectal plate, the elongated marrow and the cerebellum. After injection of gadolinium there is contrast enhancement of the mammillary bodies, the tectal plate, the thalami and the periaqueductal area.

In the late phase, there is diffuse cerebral atrophy, attrophy of the mammillary bodies and the vermis. Restriction of $\mathrm{ADC}$ is a witness of the irreversibility of the lesions. 
The presumptive diagnosis can be confirmed by measuring the blood concentration of thiamine or its phosphate derivatives (monophosphate or diphosphate).

With this clinical presentation, many diagnoses can be mentioned, in particular ischemic or hemorrhagic cerebrovascular accident. In the context of alcoholism, acute alcohol intoxication or a withdrawal syndrome may be mentioned.

On MRI, peri-ventricular involvement and medial thalamic involvement are not entirely specific for Gayet-Wernicke encephalopathy, they can be found in demyelinating pathologies of inflammatory or infectious diseases such as Behçet's disease or centro or extra pontic myelinolysis.

In the first case report, given the clinical context and the radiological presentation, extra pontic myelinolysis was the main differential diagnosis that we had considered. However, the neurological disturbances set days after the correction of the fluid and electrolyte disturbances helped to eliminate this diagnosis.

The Gayet-Wernicke encephalopathy is considered a medical emergency, treatment should never be delayed by vitamin dosages. The European Federation of Neurological Societies recommends the administration of thiamine, preferably intravenously before any administration of glucose and resumption of a normal diet, until clinical signs improve [7].

The prognosis of Gayet-Wernicke encephalopathy is extremely variable, and depends mainly on the early treatment. There is a rapid regression of abnormalities on MRI after treatment with secondary dilation of the 3rd ventricle and Sylvius aqueduct, not clinically correlated [2]. In our patients, the clinical and radiological outcome was partially favorable given the persistence of episodic retrograde memory disorder and some hypersignals on the follow up MRI. Mortality occurs in 17-20\% of cases.

\section{CONCLUSION}

Gayet-Wernicke encephalopathy is a rare disease. The triad associating ophthalmoplegia, mental confusion and ataxia is very evocative. In case of an incomplete triad, the MRI can show very suggestive abnormalities confirming the diagnosis, especially in the acute phase. However, a normal MRI does not rule out the diagnosis.

\section{REFERENCES}

1. DialloI ,BouryGningSet Al. Encéphalopathie de Gayet-Wernicke compliquant des vomissements sur terrain de néoplasie colique. Pan AfricanMedical Journal. 2015; 21:179.

2. Farquet V, Alvarez V, Biselx S et Coutaz M. Du déficit en thiamine àl'encéphalopathie de GayetWernicke, pathologie méconnue.Rev Med Suisse. $2017 ; 13: 382-4$

3. Zuccoli G, Pipitone N. NeuroimagingFindings in Acute Wernicke's Encephalopathy. Review of the Literature. AJR. 2009; 192:501-508.

4. Young-Chul Jung, Chanraud S, and Edith V. Neuroimaging of Wernicke'sEncephalopathy andKorsakoff's Syndrome. NeuropsycholRev. 2012 June; 22(2): 170-180.

5. Lenz V, Vargas M I. Apport de l'IRM dans l'exploration de l'encéphalopathie de GayetWernicke. Journal of Neuroradiology, septembre. 2002 ; Vol 29, $\mathrm{N}^{\circ} 3.153-160$.

6. Ammouri W, Harmouche H. Gayet -Wernicke encephalopathy in non alcoholic patients: A serious complication. J Rare Dis Res\&Treatment. 2016, 1(2): 59-63.

7. Galvin R, Brathen G, Ivashynka A, Hillbom M. EFNS guidelines for diagnosis, therapy and prevention of Wernicke encephalopathy. Eur J Neurol. 2010;17(12):1408-18. 